\title{
Vocal Music Therapy for Chronic Pain: A Mixed Methods Feasibility Study
}

\author{
Ming Yuan Low, MA, MT-BC, ${ }^{1}$ Clarissa Lacson, MA, MT-BC, ${ }^{1}$ Fengqing Zhang, $\mathrm{PhD}^{2}$ \\ Amy Kesslick, MA, MT-BC, LPC, ${ }^{3}$ and Joke Bradt, PhD, MT-BC ${ }^{1}$
}

\begin{abstract}
Objective: The purpose of this study was to determine the feasibility and preliminary effects of a vocal music therapy (VMT) program on chronic pain management.

Design: A mixed methods intervention design was used in which qualitative data were embedded within a randomized controlled trial.

Setting: An urban nurse-management health center on the East Coast of the United States.

Subjects: Participants $(N=43)$ were predominantly Black $(79 \%)$ and female $(76.7 \%)$ with an average pain duration of 10 years.

Intervention: Participants were randomly allocated to a 12-week VMT program or a waitlist control.

Outcome measures: We tracked consent rate (percentage of participants enrolled out of total number screened), attrition rate, and treatment adherence. We used PROMIS ${ }^{\circledR}$ (Patient Reported Outcomes Measurement Information System) tools to measure pain interference, pain-related self-efficacy, pain intensity, depression, anxiety, positive effect, and well-being, ability to participate in social activities, and satisfaction with social roles at baseline and week 12. VMT participants also completed the Patient Global Impression of Change Scale. We conducted semistructured interviews to better understand participants' experience of the intervention.

Results: The consent rate was $56 \%$. The attrition rate was $23 \%$. Large treatment effects (partial eta squared) were obtained for self-efficacy $(0.20)$, depression (0.26), and ability to participate in social activities (0.24). Medium effects were found for pain intensity $(0.10)$, anxiety $(0.06)$, positive effect, and well-being $(0.06)$, and small effects for pain interference $(0.03)$ and satisfaction with social roles $(0.03)$. On average, participants felt moderately better after completion of the VMT program $(M=4.93$, standard deviation=1.98). Qualitative findings suggest that VMT resulted in better self-management of pain, enhanced psychological well-being, and stronger social and spiritual connections.

Conclusions: Recruitment into the 12-week program was challenging, but quantitative and qualitative findings suggest significant benefits of VMT for chronic pain management.
\end{abstract}

Keywords: music therapy, pain management, clinical trials

\section{Introduction}

C HRONIC PAIN IS A SIGNIFICANT public health problem amounting to an annual health care expense of approximately half a trillion dollars in the United States alone. ${ }^{1}$ In 2016, the Centers for Disease Control and Prevention issued recommendations to move away from opioids and instead use nonpharmacological therapies for the treatment of chronic pain. ${ }^{2}$ The use of music for the management of chronic pain is increasingly

Departments of ${ }^{1}$ Creative Arts Therapies and ${ }^{2}$ Psychology, Drexel University, Philadelphia, PA.

${ }^{3}$ Stephen and Sandra Sheller 11th Street Family Health Services, Drexel University, Philadelphia, PA.

(c) Ming Yuan Low, et al. 2019; Published by Mary Ann Liebert, Inc. This Open Access article is distributed under the terms of the Creative Commons Attribution Noncommercial License (http://creativecommons.org/licenses/by-nc/4.0/) which permits any noncommercial use, distribution, and reproduction in any medium, provided the original author(s) and the source are cited. 
gaining interest, but more efficacy research is needed to encourage health care providers to recommend its use to patients. 3,4

To date, research on the use of music for pain has focused primarily on listening to prerecorded music for acute pain management with reported treatment benefits for reducing pain intensity and opioid requirements. ${ }^{5,6} \mathrm{~A}_{\text {recent review }}{ }^{3}$ on the impact of music on chronic pain reported a moderate effect size for pain (standardized mean difference $=0.60$ ), but results were inconsistent across studies $\left(I^{2}=60 \%\right)$. The majority of the studies (11/14) in this review employed listening to prerecorded music; one study used choir singing, and two studies used listening to live music.

The pain-reducing effects of music are often attributed to music's ability to distract and relax. However, chronic pain is a complex phenomenon that affects individuals physically, mentally, socially, and spiritually and its management, therefore, requires interventions that go beyond cognitive distraction. ${ }^{7}$ Therefore, we developed a vocal music therapy (VMT) treatment program that addresses biopsychosocial components of chronic pain management. ${ }^{8}$

Music therapy is the clinical use of music interventions to help clients optimize their health within a therapeutic relationship with a board-certified music therapist. ${ }^{9,10}$ The VMT group sessions use toning (i.e., singing of elongated vowels) and humming, music-guided breathing, group singing, vocal improvisations, verbal processing of emotions and thoughts evoked by the music experiences, as well as psychoeducation about how music can address biopsychosocial factors that impact chronic pain management. We briefly summarize here the intervention's theoretical framework, but readers are referred to Bradt et al. ${ }^{8}$ for a more in-depth discussion.

On a bioneurological level, music listening and music making activate brain areas involved with reward, emotion, and arousal such as the nucleus accumbens, amygdala, anterior insula, cingulate cortex, orbitofrontal cortex, and mediodorsal thalamus, through which affective and cognitive modulation of pain can be achieved. ${ }^{11-13}$ On a psychological level, toning and humming are used to help enhance body awareness, promote a positive connection with one's body, and facilitate relaxation. Since people with chronic pain often try to disconnect from their body to "escape" the pain, these are considered important mechanisms in chronic pain management. ${ }^{14}$

Singing and active music making also help facilitate emotional expression; emotional expressivity has been shown to improve a sense of well-being and self-reliance in people with chronic pain. ${ }^{15}$ Finally, group music making facilitates social inclusion and a sense of belonging. ${ }^{16,17}$ Because people with chronic pain often feel isolated, this is an important aspect of the VMT group.

The VMT program was initially tested as an 8-week program. ${ }^{8}$ Study findings were promising with large and moderate effect sizes for pain-related self-efficacy and pain interference, respectively, but participants unanimously agreed that a longer program was desirable. Yet, concerns were raised by health care providers at the study site about the feasibility to recruit people with chronic pain to a lengthier program. Therefore, the purpose of this mixed methods feasibility study was to (1) determine the feasibility of a 12-week VMT protocol; (2) provide estimates of effect for core outcomes in chronic pain management; and (3) obtain qualitative data about participant experiences of the VMT program.

\section{Materials and Methods}

\section{Study design}

We employed a mixed methods intervention design, ${ }^{18}$ in which qualitative data (i.e., semistructured interviews) were embedded within a randomized controlled trial. Participants were randomized to the VMT or waitlist control (WLC) treatment arm using a computer-generated list of random numbers. Allocation concealment was achieved through the use of sequentially numbered, opaque, sealed envelopes. Since self-report measures were used for all outcomes, outcome assessment could not be blinded as participants were aware of their treatment allocation. However, the statistician was blinded to group assignments (Fig. 1).

\section{Participants}

Participants were recruited from an urban nurse-managed health center that predominantly serves inner-city, lowincome African Americans. Eligibility criteria are included in Table 1. Participant demographic and clinical characteristics at baseline are presented in Table 2. The majority of the participants were female $(76.7 \%)$, were black $(79 \%)$, were on disability leave $(60.5 \%)$, and had an average pain duration of 10 years. No significant between-group differences were present at baseline. The study was approved by an Institutional Review Board. Informed consent was obtained from all participants. We recruited participants in three waves. In each wave, participants were randomly assigned to VMT or WLC. WLC participants were invited to participate in the VMT intervention after completion of the outcome measures at the end of the waitlist period.

\section{Interventions}

Vocal music therapy. Participants in the VMT treatment program received twelve 90-min weekly group therapy sessions (four to six participants). Sessions were led by a board-certified music therapist. The VMT sessions followed a similar structure, but were each focused on a different topic related to music-based pain management (Table 3).

After a brief music-guided deep breathing exercise and verbal check-in, the music therapist led the participants into toning (i.e., singing elongated vowels) and humming experiences. Using the voice in this manner can help facilitate greater body awareness and promote relaxation. The group then talked about somatic sensations experienced during the breathing and toning exercises.

The session then moved into vocal music improvisations. Percussion instruments were often added, resulting in energetic music making. These improvisations provided opportunities for emotional expression. Furthermore, group music making enabled participants to relate to others and share some of their struggles in novel ways. Verbal processing after the improvisation often evolved into additional improvisations focused on the main ideas of the group discussion.

Throughout the sessions, psychoeducation was provided about how music can address biological (e.g., music 


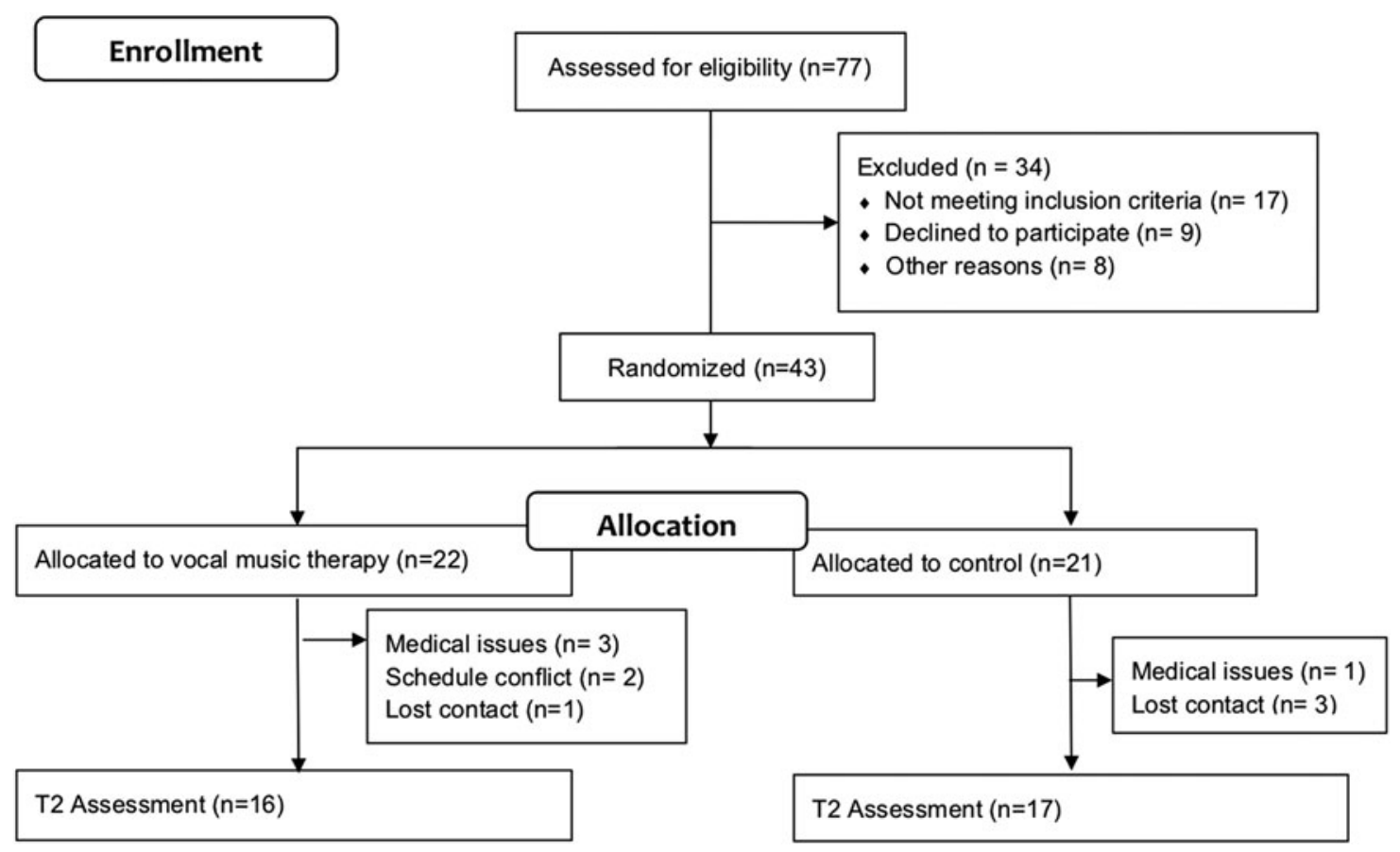

FIG. 1. Participant flow chart.

stimulates dopaminergic activity resulting in improved mood), psychological (e.g., song lyrics can help validate one's feelings), and social (e.g., group music making creates a sense of belonging) factors that play an important role in chronic pain management. Education about why and how music can address pain management can help with transferability of skills and knowledge outside of the session room and equips participants to explain to family and friends how music-based self-management techniques help them with their pain.

Each session ended with singing a song listed by one of the group members during intake. Participants were asked to underline a lyric that was particularly meaningful to them and could possibly be a source of emotional support during the week. The therapist then facilitated a discussion about the meaning of the selected lyrics. ${ }^{8}$ The music therapist was trained by J.B. using a treatment manual. Each session was recorded and reviewed by J.B. to ensure treatment fidelity.

The original protocol tested in a previous study consisted of eight 60 -min sessions. The 12-week protocol was very similar to the 8-week protocol, except that (1) the longer

Table 1. Study Eligibility Criteria

\begin{tabular}{|c|c|}
\hline Inclusion criteria & Exclusion criteria \\
\hline $\begin{array}{l}\text { English-speaking } \\
\text { adults }\end{array}$ & $\begin{array}{l}\text { Moderate to profound auditory } \\
\text { deficits }\end{array}$ \\
\hline Age 18 or older & $\begin{array}{l}\text { Severe progressive medical or } \\
\text { neurological comorbidities }\end{array}$ \\
\hline $\begin{array}{l}\text { Chronic pain } \\
\text { for } \geq 3 \text { months }\end{array}$ & $\begin{array}{l}\text { Severe psychiatric disorder } \\
\text { Cognitive impairment }\end{array}$ \\
\hline $\begin{array}{l}\text { Pain impact score } \\
\text { of } \geq 27 \text { (moderate } \\
\text { impact) })^{\mathrm{a}}\end{array}$ & $\begin{array}{l}\text { Current alcohol or drug problem } \\
\text { Currently receiving music therapy } \\
\text { services }\end{array}$ \\
\hline
\end{tabular}

\footnotetext{
${ }^{\mathrm{a}}$ Impact score items derived from Deyo et al. ${ }^{35}$
}

session length allowed for more time for each music experience and group processing and (2) the longer program length allowed for review sessions to revisit insights and music-based pain management skills gained. The VMT treatment manual will be published in the near future.

Waitlist control. Participants in the WLC group received care as usual at the health center. At the center, chronic pain management typically consists of pharmacological treatment and physical therapy services. Additional complementary services are available, including yoga and fitness classes.

\section{Outcome measures}

To determine feasibility, we tracked the following: (1) consent rate (percentage of participants enrolled out of total number screened); (2) attrition rate; and (3) treatment compliance (number of sessions attended). To measure the effects of the intervention, we used the Patient Reported Outcomes Measurement Information System (PROMIS $\left.{ }^{\circledR}\right)^{19}$ short forms (SF) to measure pain interference (SF-6b), pain-related self-efficacy (SF-6), pain intensity (SF-3a), depression (SF-4a), anxiety (SF-4a), positive effect, and well-being (SF), ability to participate in social activities (SF4a), and satisfaction with social roles and activities (SF-4a). Finally, participants rated their perception of improvement using the Patient Global Impression of Change Scale (PGIC). ${ }^{20}$

Measurements were administered at baseline and week 12 . After completion of the week 12 measurements, participants (including WLC participants who opted to receive VMT after the WLC period) were invited to participate in a semistructured interview aimed at better understanding their experience of the intervention (Appendix 1). Measurements and interviews were administered by research assistants. 
Table 2. Sociodemographic and Clinical Characteristics of Study Participants at Baseline

\begin{tabular}{|c|c|c|c|}
\hline Characteristic & Music therapy $(\mathrm{n}=22)$ & Waitlist control $(\mathrm{n}=21)$ & $\mathrm{p}$ \\
\hline Age, years, mean (SD) & $48.76(9.95)$ & $51.38(16.87)$ & 0.12 \\
\hline Gender, female, $n(\%)$ & $16(72.73)$ & $17(80.95)$ & 0.45 \\
\hline Race, $n(\%)$ & & & 0.20 \\
\hline Black & $18(81.82)$ & $16(76.19)$ & \\
\hline Caucasian & $3(13.64)$ & $0(0)$ & \\
\hline Asian & $1(4.5)$ & $0(0)$ & \\
\hline American Indian or Alaska Native & $0(0)$ & $1(4.8)$ & \\
\hline Multiracial & $0(0)$ & $4(19.05)$ & \\
\hline Employment, $n(\%)$ & & & 1.0 \\
\hline Employed & $3(13.63)$ & $2(9.52)$ & \\
\hline Unemployed & $4(18.18)$ & $3(14.29)$ & \\
\hline Retired & $1(4.5)$ & $2(9.52)$ & \\
\hline On disability & $13(59.1)$ & $13(61.9)$ & \\
\hline Duration of pain, years, mean (SD) & $9.43(7.02)$ & $10.43(11.13)$ & 0.68 \\
\hline Pain diagnosis, ${ }^{\mathrm{a}} n(\%)$ & & & 0.71 \\
\hline Arthritis & $8(36.36)$ & $10(47.62)$ & \\
\hline Degenerative disc/spinal stenosis & $2(9.09)$ & $3(14.29)$ & \\
\hline Neuropathy & $3(13.63)$ & $1(4.76)$ & \\
\hline Fibromyalgia & $2(9.09)$ & $2(9.52)$ & \\
\hline Pain impact score & $37.40(6.64)$ & $39.19(6.39)$ & 0.36 \\
\hline Prior music performance experience, ${ }^{\mathrm{b}} n(\%)$ & & & 0.75 \\
\hline Yes & $13(59.1)$ & $11(52.38)$ & \\
\hline No & $8(38.10)$ & $10(47.62)$ & \\
\hline
\end{tabular}

${ }^{\mathrm{a}}$ Most commonly reported pain diagnoses.

${ }^{\mathrm{b}}$ Having played an instrument or sung in a choir.

$\mathrm{SD}$, standard deviation.

\section{Data analysis}

Quantitative data. For each of the outcome variables, we compared the average difference between the VMT and WLC conditions in improvements of the outcome from baseline to week 12. $T$-scores were used for all PROMIS tools. ${ }^{21}$ Raw scores were used for the PGIC. Due to the small sample size, mean difference of improvement from baseline to week 12 between the two conditions and the $95 \%$ confidence interval (CI) was reported and used for inference. In addition, we compared the improvement in the outcomes between the two conditions controlling for baseline values. Partial eta squared from ANCOVA was used to quantify the effect size and

TABle 3. Session Topics

\begin{tabular}{cl}
$\begin{array}{l}\text { Session } \\
\text { number }\end{array}$ & \multicolumn{1}{c}{ Session topic } \\
\hline 1 & Introduction and rapport building \\
2 & Music making to enhance body awareness \\
3 & $\begin{array}{l}\text { Music-based techniques to promote self-care } \\
\text { and acceptance }\end{array}$ \\
4 & Music-based self-management of pain and stress \\
5 & Music as motivator for physical activity \\
6 & Review session: Review skills learned/insights \\
7 & gained to date \\
8 & Music as a source of strength and inspiration \\
9 & Emotional expressivity through music \\
10 & Mushancing social support through music \\
11 & Develop plan for maintenance of music-based \\
12 & Skills \\
\hline
\end{tabular}

was interpreted as small (0.01), moderate (0.06), and large $(0.14) .^{22,23}$ Given the limited sample size, we based our inference on effect sizes. ${ }^{24,25}$

Qualitative data. The transcripts of the interviews were imported into MAXQDA $11^{26}$ and analyzed by two coders (M.Y.L. and C.L.) to ensure scientific rigor. We used theoretical thematic analysis procedures as outlined by Braun and Clarke. ${ }^{27}$ Coding was based on a semantic approach in which codes are derived from "the explicit meaning of the data and the analyst is not looking for anything beyond what a participant has said"27 (p. 84). After codes were agreed upon by both coders, they were organized into categories. These were presented to J.B. for input and were compared against the text excerpts associated with the codes for verification. The categories were then organized into broader themes. After final categories and themes were agreed upon, definitions for the categories and themes were developed.

\section{Results}

Feasibility

The consent rate was $56 \%$. Of the 43 participants who completed the baseline, 33 completed the postintervention measures. This represents an attrition rate of $23 \%$ (Fig. 1). Of the VMT participants, nine participants attended nine or more sessions. Failure to attend a session was mainly due to childcare issues, family emergencies, bad weather, health issues, and traveling.

\section{Preliminary efficacy}

Table 3 details the mean change scores (baseline to week 12) and standard deviations (SDs) for each group as well as 
the effect sizes. There was a large treatment effect of VMT for pain-related self-efficacy, depression, and ability to participate in social activities. The 95\% CIs associated with these large effect sizes suggest that these findings were statistically significant. Medium treatment effects were found for pain intensity, anxiety, and positive affect and wellbeing, and small effect sizes for pain interference and satisfaction with social roles. The $95 \%$ CIs of these medium and small effect sizes suggest that these were not statistically significant. On average, PGIC scores $(M=4.93, \mathrm{SD}=$ 1.98) suggest that participants felt moderately better after completion of the VMT program (Table 4).

\section{Qualitative results}

A total of 25 participants took part in the semistructured interviews. All participants reported that the VMT sessions were beneficial in helping them manage their pain inside and outside of sessions (Theme 1, Pain Management). One participant remarked, "Every time I play the instruments, it helped me with my pain [...] That drum playing changed my pain in some kinda way. 'Cuz I didn't have it [pain] once I stopped doing the drums." Participants shared that they used VMT strategies to assist them with their daily activities and chores or for motivation in the morning: "I wasn't feeling too good this morning. I turned on some music and it took my mind off of that feeling. I was able to get dressed on time and I made it here on time."

Some participants reported using music-based skills as alternatives to their pain medication as the music helped to soothe the pain and refocus their attention. Other participants stated that the music made their pain "go away": "That day I was having a lot of pain. We started singing and [...] it just went away." One person commented how purposefully music listening helped them with daily activities: "There are certain songs I like, I can get into the rhythm of them. I just focus on the music part, and it gave me a rhythm. As long as I was listening to the music when I was working, I was able to keep going."

Many participants reported enhanced psychological wellbeing in response to the VMT experiences and the psychoeducation about how music can address different factors that influence their pain (Theme 2, Improved Psychological Well-being). Participants shared that the VMT program led to (1) better understanding of the contribution of stress and other emotions to their pain, (2) greater awareness of the presence of stress and uncomfortable emotions, and (3) learning new music-based skills to help deal with mental states that exacerbated their pain. One participant shared the following: "I have step-children that I take care of. It can become overwhelming. [Music] helps me just take that moment to woosh (sic) and $[\ldots]$ release that negativity so they don't see that and feel that."

Some participants told us that they are now more intentional with their use of music and that they create playlists for specific purposes. Participants also emphasized that learning to be kinder to oneself, achieving mindfulness, and understanding who they are were important skills gained from the program. One person shared that "keeps you away from that self-blame-because that [self-blame] adds to the pain." A large number of comments referred to feeling empowered to prioritize one's mental health and physical needs over demands by others and seeking out things in life that bring joy. Participants also appreciated that attending the program was a form of self-care as expressed by one participant: "It made me feel like I'm doing something for myself."

The third and final theme (Developing Meaningful Connections) relates to music aiding in facilitating deeper connections with one's spirituality and stronger bonds with others. One participant commented, "The spiritual aspect of music and the emotional feelings that I got from just beating on the drum, or playing the tambourine, or that ocean drum!"

Many participants reminisced fondly about the bonds among the group members that were created through the VMT program. One participant said, "It was wonderful because the group started out with everybody was in their little shell. [...] And on the recording [excerpts from sessions] that we heard after the group, we just heard our story, and like how amazing how everybody developed. And we became united. We became a family." Another participant appreciated the accepting and nonjudgmental environment the group provided, "With friends or family or romantic partners or even doctors, sometimes struggling so much to explain intermittent, invisible chronic pain to the point of disability $[\ldots]$ Hearing and being understood and sharing just felt really comfortable and in a way that I had not experienced." Participants shared that these bonds continued outside of the sessions (Table 5).

Table 4. Change in $T$-Scores, Mean Difference and Effect Size

\begin{tabular}{|c|c|c|c|c|}
\hline \multirow[b]{2}{*}{ Outcome } & \multicolumn{2}{|c|}{ Change score $(S D)^{\mathrm{a}}$} & \multirow[b]{2}{*}{$M D(95 \% C I)^{\mathrm{b}}$} & \multirow[b]{2}{*}{ Effect size $\left(\eta_{p}{ }^{2}\right)$} \\
\hline & $V M T$ & $W L C$ & & \\
\hline Pain-related self-efficacy & $4.84(5.14)$ & $-0.26(4.76)$ & $5.10(1.52$ to 8.68$)$ & 0.20 \\
\hline Pain interference & $-2.46(5.06)$ & $-0.45(3.52)$ & $-2.01(-5.17$ to 1.15$)$ & 0.03 \\
\hline Pain intensity & $-5.7(7.24)$ & $-1.86(4.47)$ & $-3.85(-8.19$ to 0.49$)$ & 0.10 \\
\hline Anxiety & $-2.42(8.55)$ & $0.39(7.32)$ & $-2.82(-8.56$ to 2.94$)$ & 0.06 \\
\hline Depression & $-4.92(4.83)$ & $2.56(7.99)$ & $-7.48(-12.25$ to -2.71$)$ & 0.26 \\
\hline Positive affect and well-being & $0.14(6.8)$ & $-2.22(5.96)$ & $2.36(-2.27$ to 6.98$)$ & 0.06 \\
\hline Ability to participate in social activities & $2.26(3.62)$ & $-2.55(6.53)$ & $4.81(0.99$ to 8.62$)$ & 0.24 \\
\hline Satisfaction with social roles & $1.59(5.82)$ & $-0.51(6.86)$ & $2.10(-2.49$ to 6.70$)$ & 0.03 \\
\hline
\end{tabular}

${ }^{\mathrm{a}}$ change from baseline to week 12 .

${ }^{\mathrm{b}}$ Ninety-five percent CI intervals that do not include the value zero suggest that the findings are statistically significant.

$\eta_{\mathrm{p}}^{2}$, partial eta squared based on ANCOVA.

$\mathrm{CI}$, confidence interval; MD, mean difference; SD, standard deviation; VMT, vocal music therapy; WLC, waitlist control. 
Table 5. Qualitative Findings

\begin{tabular}{ll}
\hline Themes and definitions & Categories and definitions \\
\hline Theme 1 Pain & Enhanced physical \\
management: & functioning: \\
The VMT program & Music engagement helps \\
offers participants & to improve activity \\
strategies to manage & levels and ability to do \\
pain in their daily lives. & chores
\end{tabular}

Reducing pain:

Music brings pain relief through its soothing qualities as well as through refocusing of attention

Achieving mindfulness: Music helps participants be more present by creating a relaxed and clearer state of mind.

\section{Empowerment:}

VMT empowers participants to prioritize self-care, helps to restore hope, and motivates to be active.

\section{Enhanced mind-body} connection:

VMT music experiences and psychoeducation help participants gain greater awareness of the impact of emotional and cognitive state on their pain management
Example quotes

But I use the soft jazz just to help with... I just sit there and listen to it and I close my eyes. And I just rub my knees [...] and when I feel like the feeling is going to be okay and I can get up and not have a limp or anything, I get up. And then, I don't have no limp. The knee don't be bothering me.

I use to could not even walk 2 or 3 blocks. I would have to like really sit down and take a breather. But it just seemed like once I came here [...] and we just got into the mood...once everything was over, I'd go home and I'm like "I'm not in no pain at all." And I never realize it and I'm like doing all this stuff (chores) in the house.

I would describe it [the music] as a de-stressor. A way to rethink, recharge your mind in a different direction and also to take your mind off the pain...I'm not going to say it can totally go away but it'll subside a little to a point where you're functional.

I liked the ocean drum. That is really soothing, and it just relaxed my whole body. I just listened to the sound of it, and it takes you to sitting on the beach (in your imagination) and just watching the waves. It was really peaceful.

When the pain begins to come, I try to hum [...] just direct my attention to something else.

A lot of the music that we've used were meditative, so it kind of allows me to broaden like, my mind and my perception... with sounds.

I do music for de-stress. And to take my mind off the pain...it's like a rethinking process... mindfulness. So when I'm mindful, first I do mindful exercises and breathing. Recognizing my own breath. My own heartbeat. And it tends to calm down.

And there's this sign of hope. That's what I liked about the songs...that they're sign of hope, they're sign of welcoming.

It helps your day to keep going. [...] you look over at the other person, and you see that their struggles might be a little different than yours. But you see how they push through it. So to me, it makes me push through it even more because I'm like, "Okay, you know...I'm gonna keep going."

Also, just learning patience with my pain and being kinder to my body about it. In the sense of, you know, I feel like for a long time it was mostly just being mad and having that energy toward whatever part of my body was not feeling good or what I couldn't do, so I think a lot of what I took from that was, you know, focusing on what I can do now, what I can do to help myself, and something like that. Yeah. I do have, I have a lot more now.

You were allowed to sing lousy or you were allowed to be off key. [...] In the group it just didn't matter. I watched people's volume - as their confidence level went up, their volume increased. Their voices were being heard.

The songs that we would sing, it really was so motivating. I felt like I was somewhere else.

And I think most of the benefit that I got directly from the sessions themselves was probably for me more related to emotional issues related with pain.

If you don't understand your feelings, how do you expect anyone else to? [...] this music program will help you learn to get to know yourself. Then, other things will follow.

When you sing, it release endorphins, so it helps to lift the mood and better manage the pain. 
TABle 5. (CONTINUEd)

\begin{tabular}{|c|c|c|}
\hline Themes and definitions & Categories and definitions & Example quotes \\
\hline \multirow{5}{*}{$\begin{array}{l}\text { Theme } 3 \text { Developing } \\
\text { meaningful connections: } \\
\text { The VMT program } \\
\text { promotes social, } \\
\text { cultural, and spiritual } \\
\text { connections }\end{array}$} & $\begin{array}{l}\text { Managing emotions: } \\
\text { Participants learned } \\
\text { music-based strategies } \\
\text { to decrease, work } \\
\text { through, and tolerate } \\
\text { stressful and emotional } \\
\text { situations. }\end{array}$ & $\begin{array}{l}\text { But it really showed me how if you really take the time, } \\
\text { music can really help with certain areas of pain. It didn't } \\
\text { take away the pain completely, but I do have issues with } \\
\text { PTSD and anxiety and things, and I get frustrated a lot, } \\
\text { especially with the pain. So it taught me how to just take } \\
\text { that time and take a moment. } \\
\text { When the therapist had us doing the humming and the } \\
\text { singing, it made you feel at peace. }\end{array}$ \\
\hline & $\begin{array}{l}\text { Universal connection: } \\
\text { music experiences } \\
\text { promote deeper } \\
\text { connections with } \\
\text { spiritual and } \\
\text { metaphysical entities. }\end{array}$ & $\begin{array}{l}\text { Well, it (music) is a gift from God. And God shows you } \\
\text { some of things that they're (peers) going through even } \\
\text { though you might have been through it all ready, but that } \\
\text { showed them how you can come out you strong. So it was } \\
\text { a touching...it (music) was like a ministry thing to } \\
\text { me...you know, within myself. } \\
\text { The spiritual because I go to church. And we express } \\
\text { ourselves through word and dance. I kept hearing his } \\
\text { name. He's the minister of music at my church. And I just } \\
\text { kept hearin' him...every time she (the music therapist) } \\
\text { would wave that water drum or whatever that thing is } \\
\text { called...or somebody would hit the... whatever instrument } \\
\text { they would have and I'm hearing him. }\end{array}$ \\
\hline & \multirow{3}{*}{$\begin{array}{l}\text { Social connections: } \\
\text { Group music } \\
\text { engagement and sharing } \\
\text { of experiences create a } \\
\text { group bond, developing } \\
\text { deep and meaningful } \\
\text { relationships based on } \\
\text { mutual support and } \\
\text { learning. }\end{array}$} & $\begin{array}{l}\text { Just being in a room with people and hearing their } \\
\text { experiences and hearing their active listening affirmations } \\
\text { of my experiences. We all probably have different kinds } \\
\text { of pain, but chronic pain is still chronic pain. Hearing and } \\
\text { being understood and sharing just felt real comfortable } \\
\text { and in a way that I had not experienced. }\end{array}$ \\
\hline & & $\begin{array}{l}\text { Being with other people that were going through similar } \\
\text { situations and some that had worse.[...] to know that you } \\
\text { weren't the only one. 'Cuz sometimes you can feel like } \\
\text { you're alone. Especially if someone else has not } \\
\text { experienced what you're feeling are... Understand where } \\
\text { you're coming from. }\end{array}$ \\
\hline & & $\begin{array}{l}\text { Meeting other people, that was really good-just sit around a } \\
\text { bunch of people where there is no arguing, no bitterness. Just } \\
\text { singing and being happy at that moment and I liked that. } \\
\text { We'd sing like we were on the choir together. We'd } \\
\text { harmonize. I was like, wow! It was good. It was a joy. }\end{array}$ \\
\hline
\end{tabular}

PTSD, posttraumatic stress disorder.

\section{Integration of quantitative and qualitative findings}

Participants' reports of using music-based pain management strategies at home aligned with the large treatment effect for self-efficacy as the self-efficacy questionnaire measured participants' beliefs that they are able to control their pain and use methods other than medication for pain relief. Given this finding, the small improvement in pain interference was surprising, especially since the 8-week VMT program resulted in a moderate effect size for this outcome. ${ }^{8}$ As for psychosocial outcomes, participants shared that they had learned to use music to address emotions that worsen their pain and that the VMT program had helped to develop stronger connections with others. This was supported by the large treatment effects for depression and participation in social activities (i.e., ability to do things with others).

The lack of improvement in satisfaction with social roles and activities (i.e., being able to do things for family and friends) is explained by the qualitative findings: participants stated that the VMT program had empowered them to set boundaries with friends and family and prioritize self-care, thus suggesting that "being able to do things for others" may not have been a desirable outcome for study participants. This makes sense given that this study included many low-income residents with complex family situations and high caregiver burden. Participants shared in the interviews that self-care involves being selective with their effort in taking care of other people.

\section{Discussion}

The purpose of this study was to determine the feasibility and preliminary effects of a 12-week VMT program on chronic pain management. Despite suggestions from participants in a prior study of an 8-week version of the VMT protocol to increase program length to 12 weeks, ${ }^{8}$ the consent rate for this study $(56 \%)$ was much lower than that obtained in the 8-week VMT study $(77 \%)$. The fact that the attrition rate in this study (23\%) was lower compared with 
Table 6. Comparison of Treatment Effects Between 8- and 12-Week Vocal Music Therapy Program

\begin{tabular}{lccccc}
\hline & \multicolumn{2}{c}{ 8-week program } & & \multicolumn{2}{c}{ 12-week program } \\
\cline { 2 - 5 } Outcome & Effect size (Cohen's d) & Interpretation & & Effect size $\left(\eta_{p}{ }^{2}\right)$ & Interpretation \\
\hline Pain-related self-efficacy & 1.09 & Large & & 0.20 & Large \\
Pain interference & 0.6 & Large & & 0.03 & Small \\
Pain intensity & 0.46 & Moderate & & 0.10 & Moderate \\
Anxiety & 0.39 & Moderate & & 0.06 & Moderate \\
Depression & 0.6 & Large & & 0.26 & Large \\
\hline
\end{tabular}

$\eta_{\mathrm{p}}{ }^{2}$, partial eta squared based on ANCOVA.

VMT, vocal music therapy.

the 8-week study (27\%) suggests that the length of program negatively impacted enrollment rates, but not participants' ability to complete the program.

Even though a higher percentage of people remained in the study in the 12-week program, treatment adherence (i.e., number of sessions attended) was better in the 8-week program $(86 \%$ of the participants attended seven to eight sessions). Yet, the interview data suggest that the VMT program was meaningful, beneficial, and highly enjoyable for participants in this study. Taken together, these findings suggest that people may be more hesitant to commit to a study or treatment program of longer duration and that regular, weekly attendance in a 3-month program may be more challenging due to accumulation of life- or healthrelated events in a longer period of time. Indeed, childcare issues, family emergencies, and health issues were frequently given as reasons for missing a session.

VMT had a large treatment effect on self-efficacy. Selfefficacy is considered a core outcome in chronic pain management as greater levels of self-efficacy have been associated with greater physical functioning, participation in physical activities, and performance satisfaction; enhanced health and work status; and decreased pain intensity in people with chronic pain. ${ }^{28}$ Large treatment effects were also found for depression and ability to participate in social activities. This was supported by the interview data that indicate participants derived a lot of joy and support from group music-making with their peers. The experience of having chronic pain may negatively impact relationships, thereby limiting social resources for pain management. Therefore, being connected to a community or support system has been identified as an important need of people with chronic pain. ${ }^{29,30}$

The treatment effects between the 8- and 12-week program were very similar, except for pain interference (Table 6). It is unclear at this time if the difference in pain interference is a function of treatment length or selection of outcome measure, as a different outcome measure was used in the 12-week study.

The qualitative findings, namely enhanced pain management, improved psychological well-being, and development of meaningful connections, align with the biopsychosocial framework that underlies the treatment protocol. Importantly, participants reported how active engagement in VMT experiences facilitated these benefits inside and outside of the session. Furthermore, creative engagement in musicmaking helps people tap into their inner playfulness and creative selves. These are important resources that, when strengthened, may facilitate resilience in the face of life's challenges. $^{31}$
Creative participation also empowers people to actively take part in their pain management, rather than feeling victimized by pain and relying on passive pain management strategies such as taking medicine. ${ }^{8}$ Research increasingly finds that active engagement in pain management enhances self-efficacy, which can lead to improved health outcomes. ${ }^{32,33}$ Participants reported that learning about how their stress and emotions are intricately linked to their physical pain, and being able to use music-based strategies outside of the sessions to holistically address these mind-body connections was an important treatment benefit. Self-management strategies that can be easily learned and used anywhere can lessen dependency on health care systems and reduce health care cost. ${ }^{34}$

This study has several limitations. The study was limited to one urban setting that serves mostly low-income African Americans. Furthermore, this study did not use an active control condition. Finally, all outcome measures were selfreport and subject to bias. Based on the promising preliminary treatment effects obtained in this as well as the prior study, future research on the VMT protocol should expand to statistically powered multisite trials that use active control conditions. Given the qualitative findings, future studies should consider including measures of spirituality, self-care, and empowerment. In addition, accurate measurement of pain interference may need to be explored further, given that participants spontaneously reported in the interviews improved ability to do chores and increased physical activity, yet, quantitatively, only a small effect size was found.

\section{Conclusions}

Both quantitative and qualitative data suggest important benefits of VMT for chronic pain management, particularly in the areas of self-efficacy, depression, and ability to participate in social activities. However, feasibility data indicate that recruitment into a 12-week VMT program for chronic pain may be challenging. Based on the feasibility comparison of the 8-week versus 12-week VMT protocol studies and the fact that both programs resulted in similar treatment estimates for core outcomes in chronic pain management, future research efforts should focus on efficacy and effectiveness testing of the 8-week version of the VMT program.

\section{Acknowledgments}

We like to express heartfelt thanks to all the people who participated in this study. We also would like to thank the staff and providers at Stephen and Sandra Sheller 11th Street Family Health Services for their enthusiasm for this study 
and their help with referrals. Special gratitude is extended to Lindsay Edwards, Director of Creative Arts Therapies, for her unwavering support for this research.

\section{Author Disclosure Statement}

No competing financial interests exist.

\section{Funding Information}

This work was supported through a cooperative agreement with the National Endowment for the Arts Research Labs program.

\section{References}

1. Institute of Medicine. Relieving Pain in America: A Blueprint for Transforming Prevention, Care, Education, and Research. Washington DC: Institute of Medicine, 2011.

2. Dowell D, Haegerich T, Chou R. CDC guideline for prescribing opioids for chronic pain: United States, 2016. MMWR Recomm Rep 2016;65:1-49.

3. Garza-Villarreal EA, Pando V, Vuust P, et al. Music-induced analgesia in chronic pain conditions: A systematic review and meta-analysis. Pain Physician 2017;20:597-610.

4. Cheever T, Taylor A, Finkelstein R, et al. NIH/Kennedy center workshop on music and the brain: Finding harmony. Neuron 2018;97:1214-1218.

5. Cepeda MS, Carr DB, Lau J, Alvarez H. Music for pain relief. Cochrane Database Syst Rev 2006;2:CD004843.

6. Lee J-H. The Effects of Music on Pain: A Review of Systematic Reviews and Meta-analysis. Philadelphia, PA: Temple University, 2015.

7. Buckenmaier C, Schoomaker E. Patients' use of active selfcare complementary and integrative medicine in their management of chronic pain symptoms. Pain Med 2014; 15(Suppl 1):S7-S8.

8. Bradt J, Norris M, Shim M, et al. Vocal music therapy for chronic pain management in inner-city African Americans: A mixed methods feasibility study. J Music Ther 2016;53: 178-206.

9. Bruscia K. Defining Music Therapy, 3rd ed. Oak Park, IL: Barcelona Publishers, 2014.

10. American Music Therapy Association. What is music therapy? 2019. Online document at: https://www.music therapy.org/about/musictherapy accessed April 19, 2019.

11. Koelsch S. Towards a neural basis of music-evoked emotions. Trends Cogn Sci 2010;14:131-137.

12. Brown S, Martinez MJ, Parsons LM. Passive music listening spontaneously engages limbic and paralimbic systems. Neuroreport 2004;15:2033-2037.

13. Navratilova E, Porreca F. Reward and motivation in pain and pain relief. Nat Neurosci 2014;17:1304-1312.

14. Price CJ, Thompson EA. Measuring dimensions of body connection: Body awareness and bodily dissociation. J Altern Complement Med 2007;13:945-953.

15. Keefe FJ, Lumley M, Anderson T, et al. Pain and emotion: New research directions. J Clin Psychol 2001;57:587-607.

16. Livesey L, Morrison I, Clift S, et al. Benefits of choral singing for social and mental well-being: Qualitative findings from a cross-national survey of choir members. J Public Ment Health 2012;11:10-26.

17. Boer D, Abubakar A. Music listening in families and peer groups: Benefits for young people's social cohesion and emotional well-being across four cultures. Front Psychol 2014;5:392.

18. Creswell JW. A Concise Introduction to Mixed Methods Research. Thousand Oaks, CA: Sage, 2015.

19. Bradt J. The voice of women suffering from chronic pain. In: Hadley S, ed. Music Therapy: Feminist Perspectives and Critiques. Gilsum, NH: Barcelona Publishers, 2006: 291-310.

20. Guy W. ECDEU Assessment Manual for Psychopharmacology. Washington, DC: ECDEU, 1976.

21. Health Measures. PROMIS Interpret Scores. 2019; Online document at: www.healthmeasures.net/score-and-interpret/ interpret-scores/promis accessed April 17, 2019.

22. Cohen J. Eta-squared and partial eta-squared in fixed factor ANOVA designs. Educ Psychol Meas 1973;33:107-112.

23. Ferguson CJ. An effect size primer: A guide for clinicians and researchers. Prof Psychol Res Pract 2009;40:532-538.

24. McCough JJ, Faraone SV. Estimating the size of treatment effects: Moving beyond P values. Psychiatry 2009;6:21-29.

25. Lee EC, Whitehead AL, Jacques RM, et al. The statistical interpretation of pilot trials: Should significance thresholds be reconsidered? BMC Med Res Methodol 2014;14:41.

26. MAXQDA. Version 11. Berlin, Germany: VERBI Software-Consult-Sozialforschung GmbH, 1989-2014.

27. Braun V, Clarke V. Using thematic analysis in psychology. Qual Res Psychol 2006;3:77-101.

28. Martinez-Calderon J, Zamora-Campos C, Navarro-Ledesma $\mathrm{S}$, et al. The role of self-efficacy on the prognosis of chronic musculoskeletal pain: A systematic review. J Pain 2018;19: 10-34.

29. Skuladottir H, Halldorsdottir S. The quest for well-being: Self-identified needs of women in chronic pain. Scand $\mathbf{J}$ Caring Sci 2011;25:81-91.

30. Smith T. "On their own": Social isolation, loneliness and chronic musculoskeletal pain in older adults. Qual Ageing Older Adults 2017;18:87.

31. Rolvsjord R. Resource-Oriented Music Therapy. Gilsum: Barcelona Publishers, 2010.

32. Stewart M, Brown JB, Donner A, et al. The impact of patient-centered care on outcomes. J Fam Pract 2000;49: 796-804.

33. Marks R, Allegrante J, Lorig K. Review and synthesis of research evidence for self-efficacy-enhancing interventions for reducing chronic disability: Implications for health education practice (Part I). Health Promot Pract 2005;61:37-43.

34. Delgado R, York A, Lee C, et al. Assessing the quality, efficacy, and effectiveness of the current evidence base of active self-care complementary and integrative medicine therapies for the management of chronic pain: A rapid evidence assessment of the literature. Pain Med 2014;15: S9-S20.

35. Deyo RA, Dworkin SF, Amtmann D, et al. Report of the NIH task force on research standards for chronic low back pain. J Pain 2014;15:569-585.

Address correspondence to: Ming Yuan Low, MA, MT-BC Department of Creative Arts Therapies Drexel University 1601 Cherry Street Philadelphia, PA 19102

E-mail:m13337@drexel.edu 


\section{Appendix 1. Interview Questions}

\section{Semistructured Interview Guide}

1. Can you describe your overall experience of participating in the 12-week music therapy program?

2. Did the music therapy sessions help you in any way?

In case of affirmative response:

- Can you tell me how it helped you?

In case of negative response:

- Can you give some specific reasons why the sessions were not helpful to you?

- Could you make some recommendations for changes to the music therapy program that would make it more useful for you?

3. For patients with affirmative response continue with the following:

What about the sessions was the most helpful to you?

If patient shares a rather general response, follow-up with the following:

- Are there any specific parts of the sessions that helped you?

4. If patient did not talk about any benefits related to his/her chronic pain thus far, ask the following:

Did the music therapy sessions help you with your pain in any way?

5. Have you been using the music-based skills you learned in the sessions at home for your pain or stress management?

- In case of negative response, ask the following: What has prevented you from using these at home?

6. Were there any aspects of the sessions that did not work well for you?

In case of affirmative response, possible follow-up questions are as follows:

- Can you explain why?

- Were there any other challenges you want to share?

7. You were given materials (e.g., lyrics of songs) during the music therapy program. Did you find these materials useful?

8. Would you recommend this program to others?

In case of affirmative response, possible follow-up question is as follows:

- How would you describe the program to them? 